\title{
Ground state and excitation of an asymmetric spin ladder model
}

\author{
Shu Chen*, H. Büttner, and J. Voit \\ Theoretische Physik 1, Universität Bayreuth, D-95440 Bayreuth, Germany
}

(November 7, 2018)

\begin{abstract}
We perform a systematic investigation on an asymmetric zig-zag spin ladder with inter-leg exchange $J_{1}$ and different exchange integrals $J_{2} \pm \delta$ on both legs. In the weak frustration limit, the spin model can be mapped to a revised double frequency sine-Gorden model by using bosonization. Renormalization group analysis shows that the Heisenberg critical point flows to an intermediatecoupling fixed point with gapless excitations and a vanishing spin velocity. When the frustration is large, a spin gap opens and a dimer ground state is realized. Fixing $J_{2}=J_{1} / 2$, we find, as a function of $\delta$, a continuous manifold of Hamiltonians with dimer product ground states, interpolating between the Majumdar-Ghosh and sawtooth spin-chain model. While the ground state is independent of the alternating next-nearest-neighbor exchange $\delta$, the gap size of excitations is found to decrease with increasing $\delta$. We also extend our study to a two-dimensional double layer model with an exactly known ground state.
\end{abstract}

\section{INTRODUCTION}

Quantum spin ladder systems have attracted much attention in the past few years ${ }^{1,2}$. Strong quantum fluctuations prevent any long range antiferromagnetic $(\mathrm{AF})$ order in quasi-one dimension. The magnetic phases of the ladder systems are rich and strongly dependent on their geometric structures. Several types of disordered "quantum spin liquid" phases are known ${ }^{1,3,4}$. Typical examples of two-leg ladders are the railroad ladder and the zigzag ladder. The railroad ladder has a singlet ground state with elementary triplet excitations (magnons) ${ }^{1}$. Depending on the ratio of the leg to rung exchange integrals, the zig-zag ladders may have gapless ground states with algebraically decaying spin correlations or spontaneously broken dimerized ground states ${ }^{5}$. The gapped dimer ground state is degenerate, and the elementary excitations are pairs of spinons. The spin ladders have been studied experimentally in compounds such as $\mathrm{SrCu}_{2} \mathrm{O}_{3}$ and $\mathrm{CuGeO}_{3}{ }^{6}$.

The two-leg zigzag ladder, which has been well investigated, is perhaps the simplest example of the frustrated spin model and highlights the role played by frustration. However, less attention has been paid on asymmetric spin ladders where the exchange integrals on both legs are different. Only the extreme case where one leg of a zig-zag ladder is missing entirely (sawtooth or $\Delta$-chain) has been solved $^{7,8}$. In this paper, we perform a systematic study of an asymmetric zig-zag spin ladder, which is a Heisenberg model defined on the structure shown in Fig. 1. In general, it is convenient to represent the zigzag ladder as a spin chain with nearest-neighbor (NN) exchange $J_{1}$ and next-nearest-neighbor (NNN) exchange $J_{2}$ corresponding to the inter-leg exchange $J_{1}$ and intra-leg exchange $J_{2}$. Equivalently, the asymmetric ladder model can be repre- sented as a chain with an alternating NNN exchange

$$
H=\sum_{l}\left\{J_{1} \mathbf{S}_{l} \cdot \mathbf{S}_{l+1}+\left[J_{2}+(-1)^{l} \delta\right] \mathbf{S}_{l} \cdot \mathbf{S}_{l+2}\right\}
$$

where $J_{1} \equiv 1$ and $J_{2} \pm \delta$ are the nearest-neighbor (NN) and alternating NNN coupling constants, respectively. The introduction of $\delta$ makes the exchanges on top and bottom legs different. $\delta=0$ is the ordinary zig-zag ladder or frustrated spin chain ${ }^{5}$ and $\delta=J_{2}$ is the extreme case with one leg completely missing. Fixing $J_{2}=J_{1} / 2$ gives an exactly solved continuous manifold of Hamiltonians ${ }^{9}$. The Majumdar-Ghosh $(\mathrm{M}-\mathrm{G})^{10}$ and the sawtooth chain ${ }^{7,8}$ are extreme cases with $\delta=0$ and $\delta=J_{2}=J_{1} / 2$ respectively.

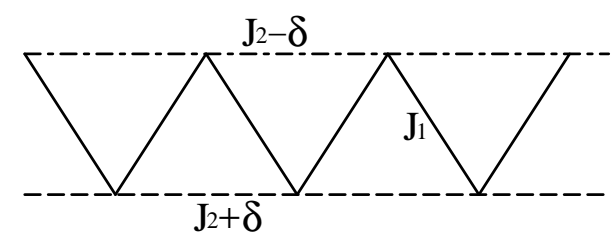

FIG. 1. The asymmetric zig-zag spin ladder with different exchanges on the top and bottom legs.

For $\delta=0$ the model (1) is well understood for general $J_{2}{ }^{5}$. Frustration due to $J_{2}$ is irrelevant when $J_{2}<$ $J_{2 c}{ }^{6,11}$, and the system renormalizes to the Heisenberg fixed point ${ }^{15}$, whose ground state is described as a spin fluid or Luttinger liquid with massless spinon excitations. As $J_{2}>J_{2 c}$, the frustration term is relevant and the ground state is doubly degenerate. Particularly, the ground state has simple product form at $J_{2}=0.5 J_{1}{ }^{10}$. The critical value of $J_{2 c}=0.2412 J_{1}$ can be determined numerically ${ }^{6,11}$. For $J_{2}>0.5 J_{1}$, quite different field theory treatments are required depending on the ratio of

*Present address: Institute for theoretical physics IV, Heinrich-Heine-Universität, D-40225 Düsseldorf, Germany 
$J_{2} / J_{1}{ }^{12,13}$.

Since relatively less is known about the asymmetric ladder, we study physical effects brought about by the leg-asymmetry. Before solving the quantum problem, we start with the classical problem which can give us an instructive insight into properties of the asymmetric spin model. In the classical limit, the ground state of this model is a Néel state for $J_{2}<J_{1} / 4$ and a spiral with a pitch $\alpha=\arccos \left(-J_{1} / 4 J_{2}\right)$ for $J_{2}>J_{1} / 4$. Both ground states and the critical ratio of exchange integrals separating them, are independent of $\delta$. The excitations may depend on $\delta$, however.

Certainly, the quantum case is much more complicated. When the asymmetric exchange interaction is introduced, some unexpected phenomena will appear. We found that the Heisenberg fixed point is no longer stable and flows to an intermediate-coupling fixed point with gapless excitations and a vanishing spin velocity. ${ }^{9}$ However, there is still reminiscence of classical results. It was found that the ground state is independent of $\delta$ when $J_{2}=J_{1} / 2$, but the excited gap is decreased by $\delta$. Part of the work on weak frustration regime has been reported in our previous letter ${ }^{9}$, and here we quantitatively study the crossover of the excitation spectrum from the symmetric M-G model to the extremely asymmetric sawtooth model in detail. An extension to a two-dimensional double layer model is also presented.

The outline of this paper is as follows. In section II, the effective low energy theory of the asymmetric ladder is derived by using bosonization. We qualitatively discuss the effect of alternating NNN interaction and compare our model with the well-known spin-Peierls model. The phase diagram of the system is discussed with the help of the renormalization group analysis. In section III, we study the asymmetric model at the special point $J_{2}=J_{1} / 2$. The ground state and excitation properties are also discussed. In section IV, we generalize our model to a two-dimensional double layer model, whose ground state is a dimer product state and the excitations are magnons. Section V contains our conclusions.

\section{EFFECTIVE LOW-ENERGY THEORY AND RENORMALIZATION GROUP ANALYSIS}

Following the general procedure of transforming a spin model to an effective model of continuum field ${ }^{14,15}$, we convert the spin Hamiltonian to a Hamiltonian of spinless fermions using Jordan-Wigner transformation, then map it to a modified Luttinger model with Umklapp and backscattering-type interactions. Using the standard dictionary of bosonization ${ }^{16,17}$, we obtain the effective boson Hamiltonian $H=H_{0}+H_{1}$ with

$$
\begin{aligned}
H_{0} & =\int d x \frac{u}{2 \pi}\left[K(\pi \Pi)^{2}+\frac{1}{K}\left(\partial_{x} \Phi\right)^{2}\right] \\
H_{1} & =\int d x\left[\frac{g_{3}}{2(\pi a)^{2}} \cos 4 \Phi+\frac{g_{1}}{\pi^{2} a}\left(\partial_{x} \Phi\right) \cos 2 \Phi\right]
\end{aligned}
$$

where $\Phi(x)$ is a bosonic phase field and $\Pi(x)$ its canonically conjugate momentum. Here $a$ is a short-distance cutoff, $g_{3} \propto 1-J_{2} / J_{2 c}$ is the Umklapp-scattering amplitude and $g_{1} \propto \delta$ is the amplitude of the alternating NNN field. The parameters $u$ and $K$ are the effective spin velocity and coupling constants which are given by

$$
u=\sqrt{\left(1+\frac{g_{4}}{2 \pi}\right)^{2}-\left(\frac{g_{2}}{2 \pi}\right)^{2}}, \quad K=\sqrt{\frac{2 \pi+g_{4}-g_{2}}{2 \pi+g_{4}+g_{2}}} .
$$

In genereal, these values are only valid near the free fermion point $(K=1)$, whereas $K=1 / 2$ is fixed by the symmetry at the isotropic point. The corresponding spin-correlation functions can be calculated from the boson representation, which gives

$$
\begin{aligned}
\left\langle S^{z}(0) S^{z}(x)\right\rangle_{0} & \sim(-1)^{x} x^{-2 K} \\
\left\langle S^{+}(0) S^{-}(x)\right\rangle_{0} & \sim(-1)^{x} x^{-\frac{1}{2 K}} .
\end{aligned}
$$

It is clear that the $\mathrm{SU}(2)$ symmetry is restored at the isotropic point with $K=1 / 2$.

For small $g_{3}$ and $g_{1}, H_{1}$ could be considered as a perturbation to $H_{0}$. Without the $g_{1}$ term, $H=H_{0}+H_{1}$ represents a standard sine-Gorden $\operatorname{model}^{5}$. The $g_{3}$ term is either marginally irrelevant, which leads to the weakcoupling Heisenberg fixed point, or relevant, which drives the system to a strong-coupling dimer state. Therefore, what we are interested in is how the alternating NNN interaction $g_{1}$ changes the physical properties of the system. Qualitative results on the influence of the new interaction $\left(g_{1}\right)$ can be obtained from scaling analysis and physical considerations.

An important information about perturbative operators is whether they are relevant, marginal or irrelevant. In general, only the most relevant perturbation is important, because the irrelevant operator will scale to zero at large lengths. We can give an approximate estimate by comparing scaling dimensions of the given operators. It follows that $e^{i \alpha \Phi(x)}$ has scaling dimension of $\frac{\alpha^{2} K}{4}$ and $\partial_{x} \Phi e^{i \alpha \Phi(x)}$ has scaling dimension of $\frac{\alpha^{2} K}{4}+1$. Therefore, the scaling dimensions of the Umklapp and the alternating NNN terms $g_{3}$ and $g_{1}$ are

$$
d_{g_{3}}=4 K, \quad d_{g_{1}}=K+1
$$

At the isotropic Heisenberg fixed point, $g_{3}$ is marginal with $d_{g_{3}}=2$, while the $g_{1}$-term with $d_{g_{1}}=3 / 2$ is relevant. We conclude that $g_{1}$ destabilizes the isotropic Heisenberg fixed point and the spin liquid ground state.

On the other hand, for $J_{2}>J_{2 c}$, the $g_{3}$ term is marginally relevant and renormalizes to a strong coupling fixed point in the long-wavelength limit. Near the strong coupling dimer fixed point, the $g_{3}$ term is much more relevant than the $g_{1}$ term. Usually (e.g. $g_{3} \rightarrow \pm \infty$ ), the boson field $\Phi(x)$ locks into a constant value with small fluctuations, and an associated excitation gap. Here, the 
constant solution $\Phi= \pm \pi / 4$ corresponds to the degenerate ground state at the strong coupling fixed point for $g_{3} \rightarrow \infty$. The standard $\cos 4 \Phi$ sine-Gordon equation has a pair of solutions of kink and antikink, which describe the elementary excitations (a pair of spinon) for the degenerate dimer phase. Even including the less relevant $g_{1}$ term, the soliton solutions will survive. However, the phase locking of $g_{1}$ term is forbidden by the $\partial_{x} \Phi$-prefactor to the $\cos (2 \Phi)$-term in $H_{1}$. In this sense, there is no standard strong coupling theory for the $g_{1}$-term.

From known results on the sawtooth chain ${ }^{7,8}$ and the M-G model ${ }^{10}$, we expect that the $g_{1}$ term, induced by the alternating NNN interaction, does not confine spinons and plays a quite different role than the dimerization by other degrees of freedom. Moreover, the difference in the size of excitation gaps in these two models implies that the $g_{1}$ term quite generally competes with the Umklapp term whereas an external NN dimerization would cooperate. As we will show, it turns out that $g_{1}$ opens no spin gap despite being a relevant perturbation of the Heisenberg fixed point. This result is also corroborated by the absence of a magnetization plateau in our model in small magnetic fields ${ }^{18}$. For an alternating NN exchange, a magnetization plateau is observed in small magnetic fields, but for alternating NNN exchange, it is only observed in high fields ${ }^{18,19}$.

To get an instructive insight, we would like to compare the alternating NNN interaction in our model with the alternating NN interaction in the well-known spin-Peierls (SP) model. In the language of field theory, the external dimerization corresponds to a relevant term $H_{s p} \propto$ $\int d x\left(g_{s p} \sin 2 \Phi\right)$. This term is always much more relevant than the $g_{3}$ term and lifts the degeneracy $(\Phi= \pm \pi / 4)$ of the ground state. With the presence of the $g_{s p}$ term, the lowest energy configuration is $-\pi / 4$ if $g_{s p}>0$ or $\pi / 4$ if $g_{s p}<0$. Since the most relevant term is $\sin 2 \Phi$, the corresponding sine-Gordon system has a pair of soliton and antisoliton solutions (excitations with $S_{z}= \pm 1$ ) as well as two breather solutions (excitations with $S_{z}=0$ ). ${ }^{20,21}$ The lowest breather is precisely degenerate with the soliton and antisoliton excitations, and they form a bound state which corresponds to $S=1$ triplet. In this case, the elementary excitation should be a spin triplet and a spin singlet, no spinons exist as elementary excitation. ${ }^{22-24}$ However, the alternating NNN interaction, $\left(\partial_{x} \Phi\right) \cos 2 \Phi$, does not lift the degenerate phases $\Phi= \pm \pi / 4$, due to the existence of the pre-factor $\partial_{x} \Phi$.

We now perform a perturbative renormalization group (RG) analysis to our model by following standard procedures. ${ }^{25-28}$ Introducing the reduced variables $y_{3}=$ $\frac{g_{3}}{\pi u}$ and $y_{1}=\frac{g_{1}}{\sqrt{2} \pi u}$, we obtain the RG equations

$$
\begin{aligned}
& \frac{d K}{d l}=-y_{3}^{2} K^{2}+y_{1}^{2} K^{4} \\
& \frac{d y_{3}}{d l}=(2-4 K) y_{3}+K^{2} y_{1}^{2} \\
& \frac{d y_{1}}{d l}=(1-K) y_{1}-4 K^{2} y_{1} y_{3}
\end{aligned}
$$

$$
\frac{d u}{d l}=-\frac{1}{2} u y_{1}^{2}(1+K) K^{2}
$$

under a change of length scale $a \rightarrow a e^{d l}$. Here we define $d l=\ln \frac{a+d a}{a}$. The RG equation for the spin velocity $u$ is a consequence of the anisotropy of the $g_{1}$-interaction in the classical 2D XY-model, i.e. its non-retarded but non-local character in the quantum field theory (3).

For $y_{1}=0$ equations (9) and (10) would not appear, thus $u$ is not renormalized. The RG equations (7) and (8) with $y_{1}=0$ describe the symmetric spin ladder system with a Kosterlitz-Thouless transition. ${ }^{5}$ Spinrotation invariant models scale along the separatrix between Néel and spin liquid phases, or along its continuation into the dimer regime. Linearizing the RG equations (7)-(10) around the isotropic Heisenberg fixed point $\left(g_{3}^{(H)}=0, g_{1}^{(H)}=0, K^{(H)}=1 / 2\right)$ and defining $\delta K=K-\frac{1}{2}$, we obtain the linearized RG equations

$$
\begin{aligned}
\frac{d \delta K}{d l} & =-\frac{1}{4} y_{3}^{2}+\frac{1}{16} y_{1}^{2}, \\
\frac{d y_{3}}{d l} & =-4 \delta K y_{3}+\frac{1}{4} y_{1}{ }^{2}, \\
\frac{d y_{1}}{d l} & =\frac{1}{2} y_{1}-y_{1} y_{3}-\delta K y_{1} .
\end{aligned}
$$

A family of solutions of the RG equations, projected on the $y_{3}-K$-plane, are shown in Fig. 2. We choose the initial value of $y_{1}$ as 0.001 and find the trajectories are not sensitive to the choice of the initial values of $y_{1}$. From the RG equations, one can directly find that there exist two intermediate fixed points given by $\left(\delta K, y_{3}, y_{1}\right)=(0.1,0.4, \pm 0.8)$. Here $y_{1}$ takes the values \pm , which reflects that our RG equations is symmetric to $y_{1}$. As shown in Fig. 2, the intermediate fixed point $\left(\delta K^{\star}, y_{3}^{\star}\right)=(0.1,0.4)$ on the plane of $y_{3}-\delta K$ is stable along the line $y_{3}=4 \delta K$ where the spin-rotation invariance is protected. For points near the intermediate fixed points, but not exactly on the line of $y_{3}=4 \delta K$, the spin-rotation invariance is broken, thus they will flow to the spin-fluid fixed point $\left(y_{3}=0\right)$ or the strong coupling fixed point $\left(y_{3} \rightarrow \infty\right)$.

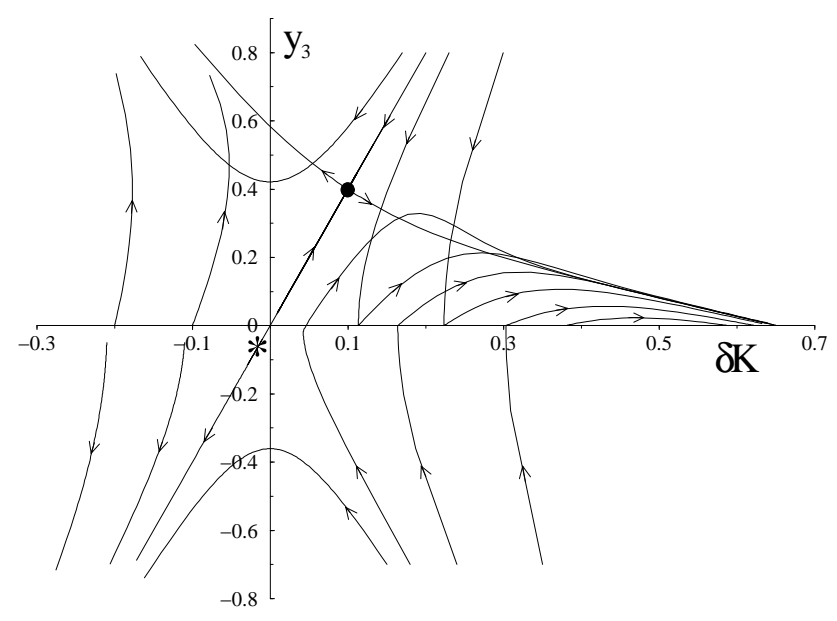


FIG. 2. The scaling trajectories for $y_{1}(l=0)=0.001$ projected on the $y_{3}-\delta K$ plane. $\delta K=K-1 / 2$, and the dot locates the new intermediate coupling fixed point. The Néel state is realized in the upper left, the dimer state in the lower left, and the spin liquid in the right part of the figure. The asterisk locates the boundary between flows to the new fixed point, and into the dimer regime.

At the fixed point, the RG equation (10) implies that the spin velocity $u^{\star}$ is renormalized. The robustness of the existence of this intermediate fixed point against higher order perturbations does not depend on the exact value of the fixed point as long as the fixed point is located on the $\mathrm{RG}$ separatrix with $1 / 2<K<\infty$. $^{9}$ The intermediate fixed point is thus described by an effective fixed-point Hamiltonian. Inverting the definitions of $y_{i}=g_{i} / \pi u$, we rewrite the effective fixed-point Hamiltonian as a product of $u^{\star}$ and a term independent of $u^{\star}$, $H^{\star}=u^{\star} \mathcal{H}\left(K^{\star}, g_{3}^{\star}, g_{1}^{\star}\right)$. Then the vanishing of $u^{\star}$ leads to a trivial fixed point Hamiltonian $H^{\star}=0$. As a result of the vanishing of the renormalized spin velocity, the elementary excitations at the fixed point, spinon and antispinon, are still gapless. The vanishing of velocity is usually interpreted as a sign of ferromagnetism, however we interpret this as our spins effectively decoupling at the lowest energy scales, i.e. a kind of asymptotic freedom in this spin-rotation invariant ladder ${ }^{9}$. Also, the numerical results of Wiessner et al. ${ }^{18}$ indicate a paramagnetic susceptibility.

When $J_{2}$ increases beyond a critical value $J_{2 c}(\delta)$ (now depending on $\delta$ ), the RG flows to a strong coupling fixed point, which corresponds to the quantum dimer phase. For $y_{1}=0.001$, this critical point is indicated in $\left(K, y_{3}\right)$ coordinates in Fig. 2 by an asterisk. For small $\delta$ and $J_{2}>J_{2 c}(\delta)$, our RG equations show that the system will remain in the universality class of the dimer solid corresponding to the strongly fixed point, however the spin gap is decreased by increasing $\delta$. Basically, the gap size $\Delta \propto \exp \left(-l_{1}\right)$ where $l_{1}$ is the length of the scaling trajectory from the initial values to the point where the most relevant perturbation is of order unity. This length is increased, and $\Delta$ therefore decreased, by the $y_{1}$ contributions to $K$ and $y_{3}$ being opposite in sign to those of $K$ and $y_{3}$ in Eqs. (7) and (8).

Recently, Sarkar and $\operatorname{Sen}^{29}$ studied the same model by using a nonlinear $\sigma$-model field theory and Abelian bosonization. However, the main discrepancy between our work and theirs ${ }^{29}$ is that we kept the bosonized operator of the alternating NNN operator and analyzed it by RG, while they just discarded it by giving an argument of the irrelevance of the operator. For an anisotropic XXZ chain, our RG result indeed shows that the $g_{1}$ operator is irrelevant ${ }^{9}$ in the meaning that it does not drive the system to a new phase, and this is consistent with that of Sarkar et. al. But the main difference lies in the question whether an intermediate fixed point exists and whether this fixed point corresponds to a phase different from a Luttinger liquid. In our previous work the argument of a vanishing spin-wave velocity plays a crucial role in the existence of such an unusual phase. If the spin-velocity does not vanish, one should explain the fixed point as a spin-liquid phase as in the $J_{1}-J_{2}$ model with the spin velocity renormalized. Our result also suggests that the quantum phase transition parameter $\left(J_{2 c} / J_{1} \simeq 0.2412\right)$ to the dimer phase is changed by the alternating NNN operator, which may be verified directly by numerical simulations like the density-matrix renormalization group. We also notice that the magnetization curve of the Heisenberg model with an additional alternating NNN operator ${ }^{18}$ gives an obvious different magnetization susceptibility from the one without it. Their susceptibility is enhanced by this new interaction with respect to an equivalent Heisenberg chain, indicating a reduced spin velocity. Furthermore, a complete scheme to deal with the alternating NNN operator should give a correct description of the induced effect not only on the weak frustration regime but also on the strong frustration regime. The omission of the $g_{1}$ term could not give any explanation why the operator shrinks the spin gap sizes in the regime of strong frustration as we will study in detail in the next section. However, our RG analysis gives a qualitative explanation of the influence of the $g_{1}$ term on spin gap sizes. Therefore, we think that the scheme of discarding the $g_{1}$ operator based on its irrelevance seems to be oversimplified. We hope that more numerical simulations will eventually be able to resolve this disagreement and study quantitatively the phase diagram of the ground state as a function of $J_{2} / J_{1}$ and $\delta$.

\section{CROSSOVER FROM M-G TO SAWTOOTH MODEL}

It is generally believed that continuum field theory cannot give a good description for the behavior of the system far away from the critical point. In the case of $J_{2}=0.5 J_{1}$, the correlations extend only to a distance of one lattice spacing, thus the continuum field description is not a good approach. As we have shown, the phase corresponding to large $J_{2}$ is the dimer phase. This is consistent with our knowledge from the models with exactly known ground states, say, Majumdar-Ghosh (M-G) model and sawtooth model. In this section, we prove that there exists a continuous manifold of Hamiltonians with dimer product ground states as long as $J_{2}=J_{1} / 2$.

We start with the asymmetric ladder model

$$
H=\sum_{l=1}^{2 N} J \mathbf{S}_{l} \cdot \mathbf{S}_{l+1}+\left[\frac{J}{2}+(-1)^{l+1} \delta\right] \mathbf{S}_{l} \cdot \mathbf{S}_{l+2} .
$$

The M-G model and sawtooth model are corresponding to $\delta=0$ and $\delta=J / 2$ respectively.

For the M-G model ${ }^{10}$, the two linearly independent ground states, say, the left and right dimer ground states, are products of nearest-neighbor singlets, respectively 


$$
\left|\Phi_{L}\right\rangle=\prod_{l=\text { odd }}[l, l+1], \quad\left|\Phi_{R}\right\rangle=\prod_{l=\text { even }}[l, l+1],
$$

where $[i, j]=\left(\alpha_{i} \beta_{j}-\beta_{i} \alpha_{j}\right) / \sqrt{2}$ denotes the singlet combination of spin $\mathrm{i}$ and $\mathrm{j}$ with the direction of dimers defined as $i \rightarrow j$. Here $\alpha_{i}$ represents the up-spin and $\beta_{i}$ the down-spin state at site $i$. $\left|\Phi_{L, R}\right\rangle$ also represent the degenerate ground states of the sawtooth model ${ }^{7,8}$. For the asymmetric ladder model, we notice that the NNN exchange alternation does not modify the product states of nearest-neighbor singlets

$$
H_{\delta}\left|\Phi_{L, R}\right\rangle=\sum_{l}(-1)^{l} \delta \mathbf{S}_{l} \cdot \mathbf{S}_{l+2}\left|\Phi_{L, R}\right\rangle=0 .
$$

This is induced by the fact that the alternating NNN couplings along the upper leg and the lower leg of the ladder cancel out each other, when they operate on $\left|\Phi_{L, R}\right\rangle$. It is obvious that $\left|\Phi_{L}\right\rangle$ and $\left|\Phi_{R}\right\rangle$ are eigenstates of the Hamiltonian (14). In fact, as we will prove, they are exactly the ground states of (14).

To see this more clearly, we rewrite the asymmetric ladder model as a sum of projection operators $P_{l}^{3 / 2}$ :

$$
H=\sum_{n=1}^{N}\left[\frac{3}{2}\left(\frac{J}{2}-\delta\right) P_{2 n-1}^{3 / 2}+\frac{3}{2}\left(\frac{J}{2}+\delta\right) P_{2 n}^{3 / 2}-\frac{3}{4} J\right] .
$$

with

$$
P_{l}^{3 / 2}=\frac{1}{3}\left[\left(\mathbf{S}_{l-1}+\mathbf{S}_{l}+\mathbf{S}_{l+1}\right)^{2}-\frac{3}{4}\right] .
$$

Here, we introduce $l$ to indicate the center position of three neighboring sites $(l-1, l, l+1)$. Such an operator is a special case of the general positive semidefinite Löwdin's projection operators ${ }^{30}$

$$
P^{S_{\max }}=\prod_{S=S_{\min }}^{S_{\max }-1} \frac{\left(\mathbf{S}_{1}+\mathbf{S}_{2}+\cdots+\mathbf{S}_{m}\right)^{2}-S(S+1)}{S_{\max }\left(S_{\max }+1\right)-S(S+1)}
$$

where $S_{\max }$ and $S_{\min }$ are the maximum and minimum values of the total spin $S$.

As long as $|\delta| \leq \frac{J}{2}$, the coefficients in Eq. (17) are non-negative. Therefore, the Hamiltonian (14) is a linear combination of projection operators with positive coefficients. Since $P_{l}^{3 / 2}$ projects a state composed of three spins, $\left(S_{l-1}, S_{l}, S_{l+1}\right)$, into a subspace of total spin $\frac{3}{2}$, its eigenvalues are 0 (if the total spin is $\frac{1}{2}$ ) and 1 (if the total spin is $\frac{3}{2}$ ). By virtue of the properties of the positive semidefinite projection operator, whose lowest eigenvalue is zero, the ground state of the Hamiltonian (17) can be constructed by choosing states with such configurations that each projection operator has the lowest eigenvalues 0 when operating on these states. It is easy to prove that $\left|\Phi_{L, R}\right\rangle$ are the exact ground states of the asymmetric ladder model (14). The ground state energy is independent of $\delta$ and given by

$$
E_{g}=-\frac{3}{4} N J
$$

There is thus an entire manifold of Hamiltonians with fixed $J_{1}=2 J_{2}$, parameterized by $\delta$, with doubly degenerate ground states of $\mathrm{NN}$-dimer product ground states $\Phi_{L, R}$. For convenience, in our following discussion we will shift our model by an energy of $E_{g}, H-E_{g} \rightarrow H$, which is equivalent in taking the ground state of the system as zero.

In the following, we consider the excited state of our system (14). The elementary excitation of the system is a pair of spinons known as the kink or antikink ${ }^{7-9}$. First results were obtained by Shastry and Sutherland for the M-G model ${ }^{31}$ within a variational ansatz. Since the ground state of (14) is independent of the alternating NNN exchange, the construction of the excited states for the M-G model can be directly extended to the asymmetric model (14). Breaking a singlet pair in the ground state would give rise to two unpaired "defect" spins. Therefore, the simplest excitation consists of a pair of spinons. The spinons can be thought as domain-walls separating different dimer ground state configurations. From symmetry consideration, the kink and antikink are identical in the M-G model, and specifically they have the same dispersions. With alternating NNN interaction, the symmetry between legs is broken, therefore some properties of kinks and antikinks are different, in particular their dispersion. However, they still survive as elementary excitations of the asymmetric spin ladder system.

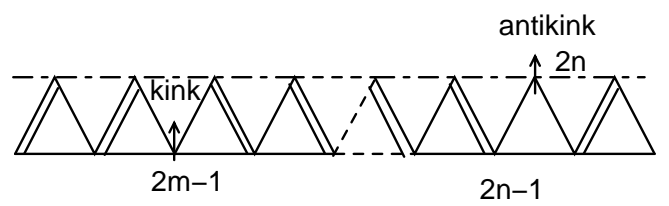

FIG. 3. The kink and antikink excitations in the asymmetric ladder model. The double lines represent singlets.

In general, we call a spinon at the odd site $2 m-1$ a kink and the other one at the even site $2 n$ an antikink (see Fig. 3). The kink and antikink always appear in pairs in a periodic system, however, a single spinon can be realized in the open boundary systems. Taking the variational wavefunction with one "defect" spin, ${ }^{31}$ one can easily obtain the spinon dispersion of the M-G model

$$
\varepsilon(k)=\frac{5}{8} J+\frac{J}{2} \cos 2 k .
$$

The energy gap $\Delta$ is therefore $J / 8$. For $\delta=J / 2$, i.e. the sawtooth model, the kink excitation is much different from the antikink excitation. As shown by D. Sen et. al. and T. Nakamura et. al. ${ }^{7,8}$, the kink (K) excitation in the sawtooth chain is exactly a single spin on odd site and dispersionless

$$
\varepsilon_{K}(k)=0 .
$$


However, an antikink propagates with an effective mass along the lattice. The antikink is not a free spin and spreads out to an extended region, because it is not a eigenstate of the local Hamiltonian. In the first approximation, the antikink $(\bar{K})$ is supposed to be a single defect spin at the even site, and the dispersion obtained by variational calculation has similar form as the spinon dispersion of the M-G model

$$
\varepsilon_{\bar{K}}(k)=\frac{5}{4} J+J \cos 2 k .
$$

with the corresponding energy gap of $J / 4$. Despite the variational nature of the dispersion, the results agree very well with exact numerical results ${ }^{32,33}$.

We will explicitly calculate the change of the gap size with increasing $\delta$. As expected, we found that the $\delta$ term changes the energy gap size of excitations, which is consistent with our conclusion obtained by the renormalization group analysis. Following D. Sen et. $\mathrm{al}^{7}$, we assume both the kink and antikink to be a 5-cluster block with spin $1 / 2$. It is known that for the M-G and sawtooth chain there is no closely bound kink-antikink pair whose energy is lower than that of a widely separated pair. Thus we can deal with the kink and antikink separately. The gap of the lowest excitation is a sum of the gaps of kink and antikink

$$
\Delta=\Delta_{K}+\Delta_{\bar{K}}
$$

where the subscripts, $K$ and $\bar{K}$, represent the kink and the antikink respectively. It should be noticed that both the M-G and sawtooth model have the same energy gap size $\Delta$ under the first approximation (1-cluster approximation). That is not true as we take more precise ncluster approximation.

Under the 5-cluster approximation, the only three linearly independent configurations that we need to consider are those shown in Fig. 4. We denote these three configurations of kink by $|2 m-1\rangle_{1},|2 m-1\rangle_{2}$ and $|2 m-1\rangle_{3}$ and the configurations of antikink by $|2 n\rangle_{1},|2 n\rangle_{2}$ and $|2 n\rangle_{3}$ respectively. Here, $(2 m-1)$ and $2 n$ denote the position of the center of 5-spin cluster corresponding to the kink and antikink. We now consider the momentum wavefunction with two variational parameters $a_{1,2}$ and $b_{1,2}$

$$
\begin{aligned}
& \left|k_{1}\right\rangle=\frac{1}{\sqrt{N}} \sum_{m} e^{i(2 m-1) k_{1}}\left[|2 m-1\rangle_{1}+a_{1}|2 m-1\rangle_{2}\right. \\
& \left.\quad+b_{1}|2 m-1\rangle_{3}\right] \\
& \left|k_{2}\right\rangle=\frac{1}{\sqrt{N}} \sum_{n} e^{i 2 n k_{2}}\left[|2 n\rangle_{1}+a_{2}|2 n\rangle_{2}\right. \\
& \left.\quad+b_{2}|2 n\rangle_{3}\right]
\end{aligned}
$$

where $k_{1}$ and $k_{2}$ are the momentum of the kink and antikink respectively.

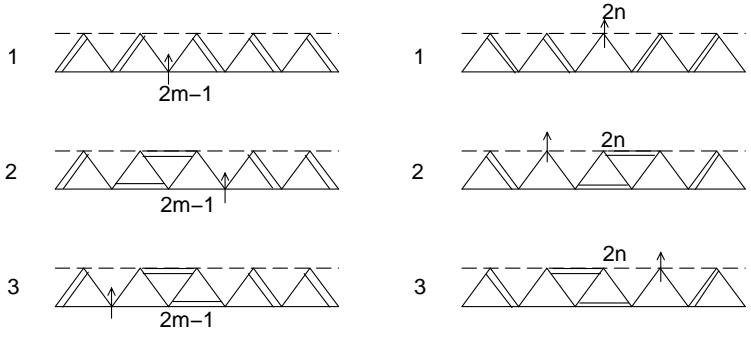

FIG. 4. The five-size cluster of the kink and the antikink.

The lowest energy is obtained by finding parameters which minimize the energy expectation

$$
\varepsilon\left(k_{1,2}\right)=\frac{\left\langle k_{1,2}|H| k_{1,2}\right\rangle}{\left\langle k_{1,2} \mid k_{1,2}\right\rangle} .
$$

Since $|2 m-1\rangle_{2}$ and $|2 m-1\rangle_{3}\left(|2 n\rangle_{2}\right.$ and $\left.|2 n\rangle_{3}\right)$ are symmetric about the site $2 m-1(2 n)$, there is no reason to discriminate between these configurations and it is reasonable to choose $a_{1,2}=b_{1,2}$.

The computation of Eq. (27) is straightforward although a little bit lengthy, we will not give the detail here but refer to the literature ${ }^{7}$. It is found that the minimum value of $\varepsilon\left(k_{1}\right)$ occurs at $k_{1}=\pi / 2$ and is given by

$$
\varepsilon\left(k_{1}=\pi / 2 ; a_{1}\right)=\frac{1}{4} \frac{J / 2\left(1+4 a_{1}^{2}\right)-\delta}{1-a_{1}+a_{1}^{2} / 2},
$$

and the minimum value of $\varepsilon\left(k_{2}\right)$ is

$$
\varepsilon\left(k_{2}=\pi / 2 ; a_{2}\right)=\frac{1}{4} \frac{J / 2\left(1+4 a_{2}^{2}\right)+\delta}{1-a_{2}+a_{2}^{2} / 2},
$$

where we take $J=1$ for convenience.

For any given value of $\delta$, the excited gap $\Delta_{K}\left(\Delta_{\bar{K}}\right)$ of a kink (an antikink) corresponds to the minimum of Eq. (28) (Eq. (29)). Our result is shown in Fig. 5, which indicates that the elementary excitation gap decreases from 0.234 in the M-G to 0.219 in the sawtooth with the increase of the coupling constant $\delta$, while the ground state energy is constant. In particular, for $\delta=1 / 2$, i.e. the sawtooth chain, Eq. (28) reduces to

$$
\varepsilon\left(k_{1}=\pi / 2 ; a_{1}\right)=\frac{1}{4} \frac{2 a_{1}^{2}}{1-a_{1}+a_{1}^{2} / 2},
$$

which has the minimum $\Delta_{K}=0$ for $a_{1}=0$, while Eq. (29) becomes

$$
\varepsilon\left(k_{2}=\pi / 2 ; a_{2}\right)=\frac{1}{4} \frac{1+2 a_{2}^{2}}{1-a_{2}+a_{2}^{2} / 2} .
$$

which has a minimum $\Delta_{\bar{K}}=0.2192$ at $a_{2}=-0.2808$. It is clear that the kink excitation is exactly dispersionless, while an antikink is still a domain wall propagating with 
an effective mass ${ }^{7,8}$. For the M-G model, $\delta=0$, Eq. (28) and Eq. (29) have the same form $\left(\Delta_{K}=\Delta_{\bar{K}}\right)$, thus

$$
\varepsilon(\pi / 2 ; a)=\frac{1}{8} \frac{1+4 a^{2}}{1-a+a^{2} / 2} .
$$

whose minimum value is 0.2344 . In these limits, the results are consistent with the known results of the M-G and the sawtooth model, as well as our qualitative conclusion obtained from the field theory.

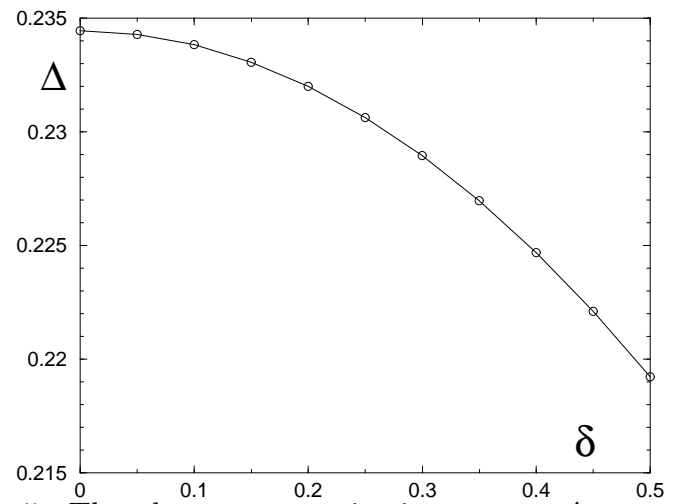

FIG. 5. The elementary excitation energy $\Delta$ versus $\delta$ at the M-G point $J_{2}=0.5$ with $\Delta=\Delta_{K}+\Delta \frac{\Delta_{K}}{\text {. }}$

If we introduce additionally an alternating NN exchange to our asymmetric model (14), the degeneracy of $\left|\Phi_{L}\right\rangle$ or $\left|\Phi_{R}\right\rangle$ will be lifted and the singlets would be pinned along the stronger NN external dimer potential. The elementary excitations are no longer separated kinks and antikinks. In the presence of an external dimer potential, a kink and an antikink separated by a distance of $l$ give rise to a region in the incorrect "ground state", which effectively produces a confining potential between the kink and antikink. This potential is proportional to the distance of $l$, thus the kink and antikink can not escape from each other and behave analogously as quarkantiquark pair. ${ }^{2-24}$ The kink-antikink bound state corresponds to a magnon with spin 1 . The interesting topic of how the confined spinons develop to magnons has been investigated by Uhrig et. al. ${ }^{34}$

\section{DOUBLE LAYER MODEL}

Recently, it was found that the 2D Shastry-Sutherland model $^{35,36}$ can be used to explain the experimentally realized material $\mathrm{SrCu}_{2}\left(\mathrm{BO}_{3}\right)_{2}$, hence such kind of model with exact dimer ground state ${ }^{37}$ attracted much attention again. In this section, we will show that the asymmetric spin ladder model can be generalized to a double layer model, whose ground state is a simple direct product of singlet dimers.

The double layer model is constructed from two coupled spin layers shown in Fig. 6, where each layer has $N \times M$ sites and couples to the other layer by the interlayer exchange interactions $J_{\perp}$ and $J_{d}$. The intra-layer exchange interactions $J^{1}$ and $J^{2}$ on top and bottom layers may have different strengths. The Hamiltonian of our model is given by

$$
\begin{aligned}
H & =\sum_{i, j=1}^{N, M} \sum_{\alpha=1}^{2} J^{\alpha}\left(\mathbf{S}_{i, j}^{\alpha} \cdot \mathbf{S}_{i, j+1}^{\alpha}+\mathbf{S}_{i, j}^{\alpha} \cdot \mathbf{S}_{i+1, j}^{\alpha}\right) \\
& +\sum_{i, j=1}^{N, M} J_{d}\left(\mathbf{S}_{i, j}^{1} \cdot \mathbf{S}_{i, j+1}^{2}+\mathbf{S}_{i, j}^{1} \cdot \mathbf{S}_{i+1, j}^{2}\right) \\
& +\sum_{i, j=1}^{N, M} J_{\perp} \mathbf{S}_{i, j}^{1} \cdot \mathbf{S}_{i, j}^{2}
\end{aligned}
$$

where the superscripts $\alpha=1,2$ denote labels of the top and bottom layers. $J_{\perp}$ is the perpendicular inter-layer exchange interaction and $J_{d}$ is the diagonal inter-layer exchange interaction. Here all the exchanges are taken to be positive. A similar model has been investigated in Ref. ${ }^{38}$, where the layer model is a direct generalization of the Bose-Gayren ladder model ${ }^{39}$. It is clear that every slice of the double layer net is just a ladder whose Hamiltonian has the same form of Eq. (14). Thus we find that the ground state of the layer model is given by a product of all perpendicular singlet pairs

$$
\Phi_{D}=\prod_{i, j=1}^{M, N} \frac{1}{\sqrt{2}}\left(\alpha_{i, j}^{1} \beta_{i, j}^{2}-\beta_{i, j}^{1} \alpha_{i, j}^{2}\right),
$$

when the condition

$$
J_{\perp}=2 J_{d}=2\left(J^{1}+J^{2}\right)
$$

is fulfilled. A rigorous proof can be made directly by representing the layer model as a sum of the projection operators as in the spin ladder case. The corresponding ground state energy is

$$
E_{g}=-\frac{3}{4} N M J_{\perp}
$$

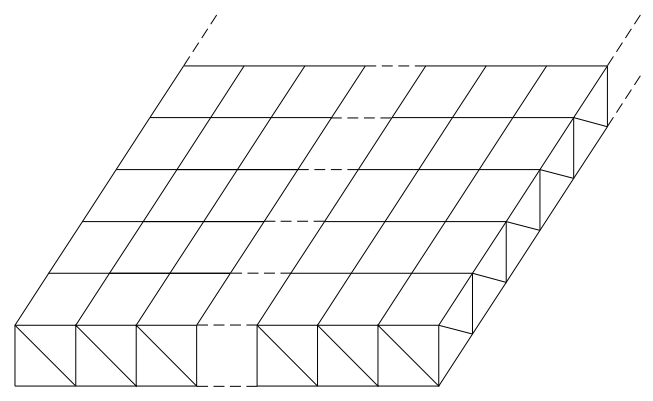

FIG. 6. The two-dimensional double layer model. 
It is obvious that the dimer product state $\Phi_{D}$ remains the ground state for $J_{\perp}>2 J_{d}$. The properties of the ground state are independent of the specific values of $J^{1}$ and $J^{2}$ as long as the constraint condition (35) is satisfied. Since the dimerized ground state is not degenerate, the lowest excitation is expected to be a triplet excitation, corresponding to breaking of a singlet bond, with a gap size proportional to $J_{\perp}$. However, the many-particle excitation spectra might be very complicated because of the effective interactions among the triplet excitations ${ }^{40}$.

\section{CONCLUSIONS}

The spin-isotropic, asymmetric zig-zag ladders are studied using the field theory method and the variational approach. When the leg exchange integrals are small compared with the NN exchange, the spin model is mapped to a revised double frequency sine-Gorden model. Renormalization group analysis shows that there are two fixed points, say, an intermediate-coupling fixed point and a strong coupling fixed point. In the weak frustration limit, the system is described by the intermediatecoupling fixed point with gapless excitations. The vanishing of spin velocity at the intermediate-coupling fixed point is likely to indicate a decoupling of spins at low energy scales. Apart from the isotropic separatrix, we find gapless spin liquid and gapped Néel states with easyplane and easy-axis anisotropy. For large frustration, a more usual dimer solid phase is realized corresponding to the strong coupling fixed point. The RG analysis also predicts that the spin gap is decreased by increasing the leg-asymmetry $\delta$. A continuous manifold of Hamiltonians with the same singlet product ground state interpolates between the Majumdar-Ghosh model and the sawtooth spin chain. Starting from the exact ground state wavefunction, we construct the variational wavefunction of the excited state and investigate the change of spin gap with the change of leg-asymmetry $\delta$. In the spirit of constructing Hamiltonian in the form of a sum of positive semidefinite projection operators, extension to the double layer model is carried out. We propose an exactly solved two-dimensional double layer model with a ground state of a product of inter-layer dimers.

\section{ACKNOWLEDGMENTS}

The authors would like to thank Prof. K. H. Mütter and Dr. M. Nakamura for interesting discussions. S. Chen would like to specially thank F. Siano and B. Han for their critical reading of the manuscript. This research was supported by Deutsche Forschungsgemeinschaft through grants no. VO436/6-2 and VO436/7-2.
${ }^{1}$ E. Dagotto and T. M. Rice, Science, 271, 618 (1996)

${ }^{2}$ E. Dagotto, Rep. Prog. phys. 62, 1525 (1999)

${ }^{3}$ A. A. Nersesyan and A. M. Tsvelik, Phys. Rev. Lett. 78, 3939 (1997); A. K. Kolezhuk and H. J. Mikeska, Phys. Rev. Lett. 80, 2709 (1998)

${ }^{4}$ Y. Wang, Phys. Rev. B. 60, 9236 (1999).

${ }^{5}$ F. D. M. Haldane, Phys. Rev. B. 25, 4925 (1982); 26, 5257 (1982).

${ }^{6}$ M. Azuma, Z. Hiroi, M. Takano, K. Ishida, and Y. Kitaoka, Phys. Rev. Lett. 73, 3463 (1994); G. Castilla, S. Chakravarty and V. J. Emery, Phys. Rev. Lett. 75, 1823 (1995).

${ }^{7}$ D. Sen, B. S. Shastry, R. E. Walstedt and R. Cava, Phys. Rev. B. 53, 6401 (1996).

8 T. Nakamura and K. Kubo, Phys. Rev. B. 53, 6393 (1996).

${ }^{9}$ S. Chen, H. Büttner and J. Voit, Phys. Rev. Lett. 87, 087205 (2001).

${ }^{10}$ C. K. Majumdar and D. K. Ghosh, J. Math. Phys. 10, 1388 (1969); 10, 1399 (1969).

${ }^{11}$ K. Okamto and K. Nomura, Phys. Lett. A. 169, 433 (1992)

12 D. Allen and D. Sénéchal, Phys. Rev. B. 51, 6394 (1995)

13 S. R. White and I. Affleck, Phys. Rev. B. 54, 9862 (1996)

14 A. Luther and I. Peschel, Phys. Rev. B. 12, 3908 (1975).

${ }^{15}$ I. Affleck, in Les Houches, Session XLIX, Fields, strings and critical phenomena, Elsevier, New York, 1989

16 J. Voit, Rep. Prog. phys. 58, 977 (1995).

17 J. V. Delft and H. Schoeller, Annalen der Physik, 4225 (1998).

${ }^{18}$ R. M. Wiessner, A. Fledderjohann, K. H. Mütter, and M. Karbach, Eur. Phys. J. B 15, 475 (2000)

19 A. Fledderjohann, C. Gerhardt, M. Karbach, K. H. Mütter, and R. Wiessner, Phys. Rev. B. 59, 991 (1999).

${ }^{20}$ R. F. Dashen et al., Phys. Rev. D. 10, 4130 (1974); Phys. Rev. D. 11, 3424 (1975).

${ }^{21}$ A. M. Tsvelik, Phys. Rev. B. 45, 486 (1992).

${ }^{22}$ I. Affleck, Cond-mat/9705127.

${ }^{23}$ A. Dobry and D. Ibaceta, Phys. Rev. B. 59, 8660 (1999)

${ }^{24}$ G. S. Uhrig and H. J. Schulz, Phys. Rev. B. 54, R9624 (1996).

${ }^{25}$ S.-T. Chui and P. A. Lee, Phys. Rev. Lett. 35, 325 (1975)

${ }^{26}$ J. M. Kosterlitz and D. J. Thouless, J. Phys. C. 6, 1181 (1973); J. M. Kosterlitz, J. Phys. C. 7, 1046 (1974).

27 T. Giamarchi and H. J. Schulz, Phys. Rev. B. 37, 325 (1988).

28 J. Voit and H. J. Schulz, Phys. Rev. B. 36, 968 (1987); Phys. Rev. B. 37, 10068 (1988).

${ }^{29}$ S. Sarkar and D. Sen, Phys. Rev. B. 65, 172408 (2002).

${ }^{30}$ P. O. Löwdin, Rev. Mod. Phys. 36, 966 (1964).

${ }^{31}$ B. S. Shastry and B. Sutherland, Phys. Rev. Lett. 47, 964 (1981).

32 E. S. Sørensen, I. Affleck, D. Augier, and D. Poilblanc, Phys. Rev. B. 58, R14701 (1998);.

${ }^{33}$ R. R. P. Singh and Zheng Weihong, Phys. Rev. B. 59, 9911 (1999).

${ }^{34}$ G. S. Uhrig, F. S. Schönfeld, M. Laukamp and E. Dagotto, Eur. Phys. J. B 7, 67 (1998).

35 S. Miyahara and K. Ueda, Phys. Rev. Lett. 82, 3701 (1999) 
${ }^{36}$ B. S. Shastry and B. Sutherland, Physica B 108, 1069 (1981); J. Richter, N.B. Ivanov and J. Schulenburg, J.Phys.: Condens. Matter 10, 3635 (1998).

37 S. Chen and H. Büttner, Eur. Phys. J. B. 29, 15 (2002).
${ }^{38}$ H. Q. Lin and J. L. Shen, J. Phys. Soc. Jpn. 69, 878 (1998).

${ }^{39}$ I. Bose and S. Gayen, Phys. Rev. B. 48, 10653 (1993).

${ }^{40}$ O. P. Sushkov and V. N. Kotov, Phys. Rev. Lett. 81, 1941 (1998). 